\title{
Oxidatively Modified Low Density Lipoprotein Is a Chemoattractant for Human T Lymphocytes
}

\author{
Heather F. McMurray, Sampath Parthasarathy, and Daniel Steinberg \\ Department of Medicine, University of California, San Diego, La Jolla, California 92093
}

\begin{abstract}
Oxidatively modified low density lipoprotein (Ox-LDL) is a known chemoattractant for monocytes. Here we demonstrate, using a modified Boyden chamber assay, that human peripheral blood $T$ lymphocytes, but not $B$ lymphocytes, also respond chemotactically to Ox-LDL, showing a threefold increase over control and an optimum response at $10 \mu \mathrm{g} / \mathrm{ml}$. Copper and endothelial cell-oxidized LDL and $\beta$-VLDL were used and gave similar results. The activity was not chemokinetic and native LDL possessed no chemoattractant activity. The chemoattractant activity was found to reside in the lipid fraction of Ox-LDL. Lysophosphatidylcholine is a major phospholipid component of Ox-LDL and is known to be chemotactic for monocytes. We show that lysophosphatidylcholine is also chemotactic for $T$ lymphocytes with a maximal fourfold increase at $10 \mu$ M. Nonmetabolizable analogues of lysophosphatidylcholine had no such chemotactic effect. Thus, Ox-LDL, by virtue of its lysophosphatidylcholine content, may contribute to the recruitment of both $T$ lymphocytes and monocytes into developing atherosclerotic lesions. (J. Clin. Invest. 1993. 92:10041008.) Key words: chemotaxis $\bullet B$ lymphocytes $\bullet$ lysophosphatidylcholine $\bullet \beta$-VLDL $\bullet$ atherosclerosis
\end{abstract}

\section{Introduction}

It has been recognized for some time that one of the earliest events in atherosclerotic lesion formation is the intimal accumulation of monocyte-derived macrophages and their subsequent development into foam cells (1-3). Only recently, however, has the presence of substantial numbers of $T$ lymphocytes been demonstrated in human fatty streaks, fibrous plaques, and advanced plaques (4-8). Within the fibrous cap of human atherosclerotic lesions, T cells can represent up to $20 \%$ of the total cell population, while B lymphocytes or natural killer cells are rare (4).

Studies in the cholesterol-fed rabbit model of atherosclerosis have been used to determine the sequence of lymphocyte and monocyte entry into the vessel wall. After only $1 \mathrm{wk}$ of cholesterol feeding, both monocytes and $\mathrm{T}$ lymphocytes were found adhering to the aortic surface and accumulating within the lesion (9). Finding that the infiltration of these two imflammatory cell types occurs at about the same time raises the

Address correspondence to Dr. Sampath Parthasarathy, UCSD Department of Medicine, 0682, 9500 Gilman Drive, La Jolla, CA 920930682.

Received for publication 2 September 1992 and in revised form 6 January 1993.

J. Clin. Invest.

(C) The American Society for Clinical Investigation, Inc. $0021-9738 / 93 / 08 / 1004 / 05 \$ 2.00$

Volume 92, August 1993, 1004-1008 possibility that they are responding to a common stimulus. While a number of chemoattractants have been suggested for the recruitment of monocytes, little is known about the factors that may influence T lymphocyte recruitment. Previous studies from this laboratory demonstrated that oxidized LDL (OxLDL) ${ }^{1}$ is a potent chemoattractant for monocytes, but not for granulocytes. It was shown that lysophosphatidylcholine (lysoPtdCho), generated during the oxidation of LDL, was the major component responsible $(10,11)$. These studies show that Ox-LDL, but not native LDL, is also a chemoattractant for circulating $\mathrm{T}$ lymphocytes. Furthermore, lyso-PtdCho was chemotactic for $T$ cells, while neither Ox-LDL nor lyso-PtdCho showed any chemotactic activity for B lymphocytes. Thus, lyso-PtdCho, generated during oxidation of LDL or from the membrane phospholipids of damaged cells, could play a pivotal role in the recruitment of both monocytes and T lymphocytes not only into atherosclerotic lesions but also to sites of tissue injury and repair generally.

\section{Methods}

Materials. Ficoll/Hypaque, BSA, HBSS, dioctanoyl, and Ham's F10 medium were from Sigma Chemical Co. (St. Louis, MO); RPMI 1640 medium was from Irvine Scientific (Santa Ana, CA); nylon wool was from Polysciences Inc. (Warrington, PA); FCS was from Hyclone Laboratories (Logan, UT); and micro-chemotaxis chambers and $5-\mu \mathrm{m}$ filters were from NeuroProbe (Cabin John, MD).

Cells. Human $\mathrm{T}$ lymphocytes were isolated from normal human peripheral blood by the method of Julius et al. (12). Briefly, mononuclear cells were recovered from the interface after Ficoll/Hypaque gradient centrifugation, resuspended in RPMI 1640/10\% human autologous serum, and plated. After a 2-h incubation, nonadherent cells were collected by rinsing plates with serum-free RPMI 1640 . This nonadherent cell population was then enriched for T cells using a nylon wool column and the nylon wool nonadherent $T$ cells were recovered. At this point adherent $B$ cells were recovered from the column with ice-cold medium. T cells were resuspended in HBSS $/ 0.2 \%$ BSA at $10 \times 10^{6}$ and $B$ cells at $2 \times 10^{6}$ cells $/ \mathrm{ml}$ for use in chemotaxis assays. The nonadherent cell suspension eluted from the column contained $88 \% \mathrm{~T}$ lymphocytes as determined by FACS ${ }^{\circledR}$ analysis using a monoclonal antibody, Leu 4, which is directed against CD3 (Becton Dickinson and Company, Mountain View, CA), 7\% B cells using goat anti-human IgG (Sigma Chemical $\mathrm{Co}$.) and $0 \%$ monocytes using an antibody against CD14 (Becton Dickinson and Company). Further FACS ${ }^{\circledR}$ analysis of isolated $\mathrm{T}$ cell populations for CD4 and CD8 positive cells showed an average $\mathrm{CD} 4 / \mathrm{CD} 8$ ratio of 0.9 . Anti-CD4 and CD8 monoclonal antibodies were purchased from Beckton Dickinson and Company.

Rabbit aortic endothelial cells were from a line established and characterized by Buonassisi and Venter (13). These cells were maintained in Ham's F10 medium/15\% FCS supplemented with epidermal growth factor at $10 \mu \mathrm{g} / \mathrm{ml}$ and used at confluence.

1. Abbreviations used in this paper: $\mathrm{CI}$, chemotactic index; lyso-PtdCho, lysophosphatidylcholine; MDA, malondialdehyde; Ox-LDL, oxidized low density lipoprotein; TBARS, thiobarbituric acid substance. 
Lipoproteins. LDL (density $=1.019-1.063 \mathrm{~g} / \mathrm{ml}$ ) was isolated by ultracentrifugation from pooled normal human plasma collected in EDTA $(1 \mathrm{mg} / \mathrm{ml})(14)$. Protein was determined by the method of Lowry et al. (15) using BSA as a standard. Copper-oxidized LDL was prepared by incubating LDL, at a concentration of $100 \mu \mathrm{g} / \mathrm{ml}$, with 10 $\mu \mathrm{M} \mathrm{Cu}^{2+}$ in $\mathrm{F} 10$ medium at $37^{\circ} \mathrm{C}$ for $24 \mathrm{~h}$. Endothelial cell-modified LDL was prepared as previously described, using cultured rabbit aortic endothelial cells (16). $\beta$-VLDL was prepared from the plasma of cholesterol-fed New Zealand White rabbits and VLDL from non-cholesterolfed rabbits as previously described (17).

Lipid peroxidation. The extent of lipid peroxidation was measured as thiobarbituric acid reactive products (TBARS) and expressed as malondialdehyde (MDA) equivalents. Both native and $\beta$-VLDL routinely had $<5 \mathrm{nmol} \mathrm{MDA} / \mathrm{mg}$ protein, while the content of MDA in Ox-LDL samples (copper and endothelial cell-oxidized LDL) ranged from 50 to $70 \mathrm{nmol} / \mathrm{mg}$ protein.

Lipid extractions. Lipid extractions were carried out according to the method of Bligh and Dyer (18). The chloroform phase was evaporated under nitrogen, the lipids were redissolved in ethanol, and aliquots were then added to HBSS for assessment in the chemotaxis assays.

Phospholipid analysis. Lipids were extracted from lipoproteins (200 $\mu \mathrm{g}$ of protein) with chloroform/methanol and the solvents were evaporated under nitrogen. The phospholipids were separated by TLC on silica gel $\mathrm{G}$ with chloroform/methanol/water, 65:35:6 (vol/vol/ vol) and visualized with iodine vapor. Phosphatidylcholine and lysoPtdCho were scraped from the plates and assayed for phosphorus content (19).

Lyso-PtdCho and analogues. 1-Palmitoyl-sn-glycerol-3-phosphocholine (lyso-PtdCho) was purchased from Sigma Chemical Co. The lyso-PtdCho analogues, 1-palmityl-propanediol-3-phosphocholine, 1palmityl-ethanediol-2-phosphocholine, and 1-palmitoyl-ethanediol-2phosphocholine were a kind gift of Dr. W. J. Baumann (Hormel Institute, Austin, MN), prepared according to Murari et al. (20) and analyzed for purity using nuclear magnetic resonance spectroscopy. Analysis by TLC using a solvent system of $\mathrm{CHCl}_{3} / \mathrm{CH}_{3} \mathrm{OH} / \mathrm{H}_{2} \mathrm{O}$ $(65: 35: 6, \mathrm{vol} / \mathrm{vol} / \mathrm{vol})$ indicated that all samples were pure and migrated as a single species. Samples were dried under $\mathrm{N}_{2}$ and resuspended in HBSS at appropriate concentrations for the chemotaxis assay.

Chemotaxis assay. Chemotaxis was measured in a 48-well modified Boyden microchemotaxis chamber (NeuroProbe) as described previously (16). Assays were carried out in triplicate, using a $5-\mu \mathrm{m} \mathrm{mem}$ brane. T lymphocytes were suspended in HBSS at $10 \times 10^{6}$ cells $/ \mathrm{ml}$ and incubated at $37^{\circ} \mathrm{C}$ in a $5 \% \mathrm{CO}_{2}$ atmosphere for $3 \mathrm{~h}$. The filters were dehydrated and stained (LeukoStat; Fisher Diagnostics, Pittsburgh, PA) and then mounted on glass slides. The cells that had migrated to the underside of the filter were counted. Seven grid areas of $0.25 \mathrm{~mm}^{2}$ each were counted per sample. Chemotactic activity is expressed as a chemotactic index (CI), defined as the number of cells migrating in response to the test substance divided by the number migrating when control medium was present in both upper and lower chambers. The mean results are shown and are, in every case, representative of two or more replicate protocols.

\section{Results}

Effect of oxidized LDL on T lymphocyte chemotaxis. Ox-LDL was prepared either by incubation with copper ion or by incubating overnight with a monolayer of endothelial cells. As shown in Fig. 1, both forms of Ox-LDL were chemotactic for T lymphocytes, with more than a threefold increase in the number of migrating cells at a concentration of $10 \mu \mathrm{g} / \mathrm{ml}$. The decrease in response at higher concentrations of Ox-LDL has ample precedent in the behavior of other chemoattractants and was not due to toxicity as determined by trypan blue exclusion after a 3-h incubation of T lymphocytes with up to $50 \mu \mathrm{g} / \mathrm{ml}$

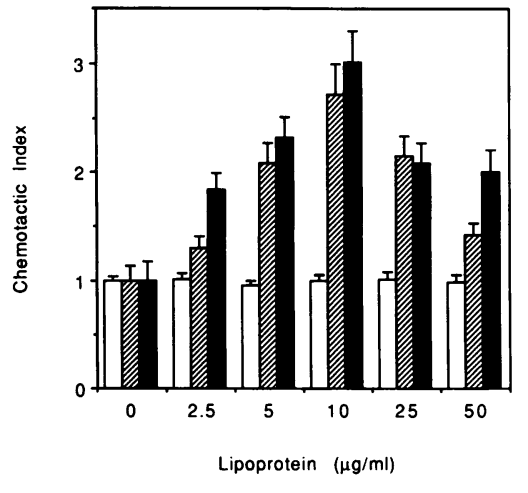

Figure 1. Human T lymphocyte chemotactic response to native (), copper-oxidized ( ( ) , and endothelial cell-oxidized ( $\bullet$ ) LDL. Samples were diluted in HBSS before testing in chemotaxis assay. Medium conditioned by endothelial cells in the absence of LDI served as the control for endothelial cell-oxidized LDL, while me-

dium without LDL served as the control for native and copper-modified LDL. The CI for each sample is expressed as mean $\pm \mathrm{SD}$ (seven fields counted). Results are representative of those from three separate experiments.

Ox-LDL. Native LDL had no chemotactic activity over the same concentration range. Checkerboard analysis was used to distinguish chemotaxis from chemokinesis and demonstrated that Ox-LDL was exclusively chemotactic in the range of 2.5$25 \mu \mathrm{g} / \mathrm{ml}$ ( Table I).

Effect of $O x-L D L$ on B lymphocyte chemotaxis. As shown in Table II, Ox-LDL in the concentration range $0-100 \mu \mathrm{g} / \mathrm{ml}$ had no significant chemoattractant activity for peripheral B lymphocytes.

The lipid components of $O x-L D L$ and lyso-PtdCho contain chemotactic activity. Unfractionated lipids extracted from OxLDL, but not from native LDL, were found to contain chemotactic activity for $\mathrm{T}$ lymphocytes, inducing the same degree of chemotactic response seen with intact Ox-LDL (data not shown). A previous report from this laboratory established that the lyso-PtdCho component of Ox-LDL was responsible for much of the ability of Ox-LDL to attract circulating monocytes (11). Consequently, lyso-PtdCho was tested using T lymphocytes as the target cells. Fig. 2 shows a fourfold increase in $T$ lymphocyte migration in the presence of 5-10 $\mu \mathrm{M}$ lyso-PtdCho. This concentration lies well below the critical micellar concentration for lyso-PtdCho in HBSS and so is not due to a simple detergent effect. Furthermore, the effective concentration of lyso-PtdCho correlates well with the estimated lyso-PtdCho content of the effective concentration of Ox-LDL. Thus, $10 \mu \mathrm{g}$ of Ox-LDL contains $\sim 2.5 \mathrm{nmol}$ of lyso-PtdCho. In contrast, PtdCho possessed no chemoattractant activity for T lymphocytes.

$\beta$-VLDL is chemotactic for $T$ lymphocytes. In the cholesterol-fed rabbit model of atherosclerosis, the major lipoprotein fraction is $\beta$-migrating very low density lipoprotein ( $\beta$-VLDL). We have previously demonstrated that $\beta$-VLDL is chemotactic for monocytes, probably by virtue of its high lyso-PtdCho content (17). The $\beta$-VLDL preparations contained, on average, $2.4 \mathrm{nmol}$ of lyso-PtdCho/ $10 \mu \mathrm{g}$ protein. Here, we show that $\beta$-VLDL is also chemotactic for T lymphocytes (Fig. 3 ). The maximal response was seen at $10 \mu \mathrm{g} / \mathrm{ml}$ and the magnitude of the response was comparable to that observed for Ox-LDL.

Extent of oxidative modification, lyso-PtdCho content, and the ability of modified LDL to stimulate $T$ cell migration. Table III clearly shows the increase in production of chemoattractant agent(s) with time during incubation of native LDL with $5 \mu \mathrm{M}$ copper acetate. The generation of chemotactic activity was 
Table I. Checkerboard Analysis of the Chemoattractant Effect of Ox-LDL on Human T Lymphocytes

\begin{tabular}{ccccr}
\hline & \multicolumn{3}{c}{ Cu Ox-LDL above filter $(\mu \mathrm{g} / \mathrm{ml})$} \\
\cline { 2 - 5 } Cu Ox LDL below filter & 0 & 2.5 & 5 & 10 \\
\hline$\mu \mathrm{g} / \mathrm{ml}$ & & & & \\
0 & $1.0 \pm 0.09$ & $0.93 \pm 0.08$ & $1.06 \pm 0.10$ & $1.02 \pm 0.10$ \\
2.5 & $1.29 \pm 0.11$ & $1.07 \pm 0.11$ & & $1.04 \pm 0.16$ \\
5 & $2.08 \pm 0.19$ & & & $1.03 \pm 0.11$ \\
10 & $2.73 \pm 0.26$ & & & $1.04 \pm 0.13$ \\
25 & $2.15 \pm 0.19$ & & & \\
\end{tabular}

The $\mathrm{CI}$ for each sample is expressed as mean $\pm \mathrm{SD}$ (seven fields counted per sample). Results are representative of those from two separate experiments.

maximal after $8 \mathrm{~h}$ when the formation of lyso-PtdCho also reached its plateau, suggesting a parallel between chemoattractant production and lyso-PtdCho formation.

Lyso-PtdCho analogues and their chemotactic activity. Previous studies have suggested the potential involvement of a phospholipase C-type reaction in the monocyte chemotactic activity of lyso-PtdCho (21). To ascertain whether metabolizable analogues of lyso-PtdCho would have effects similar to those of lyso-PtdCho itself, several analogues of lyso-PtdCho were studied: palmitoyl-ethanediol-2-phosphocholine, palmityl-ethanediol-2-phosphocholine, and palmityl-propanediol-2phosphocholine. The first is a metabolizable analogue; the latter two having the palmitate in ether linkage rather than ester linkage are not metabolizable. Fig. 4 clearly shows that only the metabolizable analogue (palmitoyl-ethanediol-2-phosphocholine) is chemotactic for T lymphocytes, showing a threefold increase in the $2.5-5 \mu \mathrm{M}$ concentration range. The increase in the number of migrating cells induced by the metabolizable analogue is equivalent to that induced by $5 \mu \mathrm{M}$ lyso-PtdCho in the same assay. The fact that only the metabolizable analogue possessed chemoattractant activity suggests that the lysoPtdCho must first be taken up by the cells before exerting its effect and provides further evidence against this being a simple detergent effect.

Table II. Ox-LDL Is Not Chemotactic for Human B Lymphocytes

\begin{tabular}{cc}
\hline Ox-LDL & $\mathrm{Cl} \pm \mathrm{SD}$ \\
\hline$\mu g / m l$ & \\
0 & $1.00 \pm 0.10$ \\
2.5 & $0.93 \pm 0.11$ \\
5 & $0.94 \pm 0.14$ \\
10 & $0.89 \pm 0.16$ \\
25 & $1.00 \pm 0.10$ \\
50 & $0.89 \pm 0.09$ \\
100 & $0.90 \pm 0.10$
\end{tabular}

The $\mathrm{CI}$ for each sample is expressed as mean $\pm \mathrm{SD}$ (seven fields counted per sample). Dioctanoyl, a protein kinase $C$ activator, was used as a positive control to ensure that the $B$ cells were capable of responding chemotactically. $100 \mu \mathrm{M}$ dioctanoyl gave a chemotactic index of $2.82 \pm 0.15$. Results are representative of those from two separate experiments.

\section{Discussion}

The demonstrated chemotactic activity of Ox-LDL for T lymphocytes suggests yet another mechanism by which Ox-LDL may influence circulating leukocytes to enter the intimal space. Native LDL had no such chemotactic activity. Furthermore, the fact that Ox-LDL was not chemotactic for isolated peripheral B lymphocytes is consistent with the observation that B lymphocytes are not found at any stage of lesion development $(4,22)$. Thus, it is entirely possible that Ox-LDL, generated in the subendothelial space, may be important in the very earliest stages of atherogenesis.

T cells persist in lesions at all stages $(6,8)$. They are especially prominent at the shoulders of more advanced lesions, in close association with macrophages, which are also present in high numbers in the shoulder regions $(4,7)$. The chemotactic effect described here may, therefore, be relevant not only in the initial stages of lesion formation but throughout the history of the lesion. IL- 1 has been demonstrated to be a chemoattractant for T cells $(23,24)$ and Ox-LDL has been shown to increase the secretion of IL-1 $(25,26)$. Thus, there could be an indirect facilitation of $T$ cell recruitment by $O x-L D L$ in addition to the direct effect demonstrated here.

It is possible that the chemotactic activity of Ox-LDL and $\beta$-VLDL for T lymphocytes could be accounted for by its content of lyso-PtdCho, in consonance with our earlier findings regarding the chemotactic activity of Ox-LDL for monocytes $(10,11)$. Furthermore, the increase in extent of modification over time paralleled with the observed increase in lyso-PtdCho

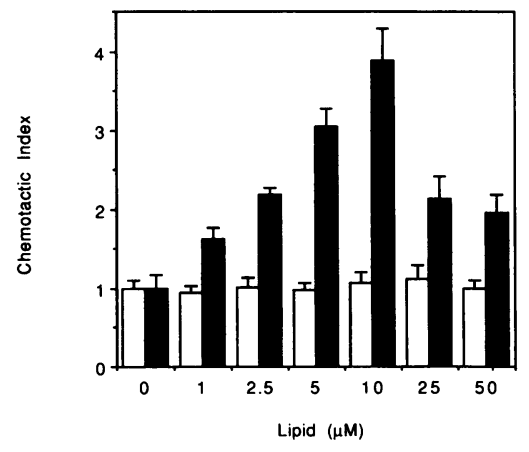

Figure 2. Human T lymphocyte chemotactic response to lysoPtdCho ( $)$ and PtdCho (ם). Both lipids were dried under nitrogen, redissolved in ethanol, and diluted in HBSS before being tested. The $\mathrm{CI}$ for each sample is expressed as mean $\pm \mathrm{SD}$ (seven fields counted). Results are representative of those from three separate experiments. 


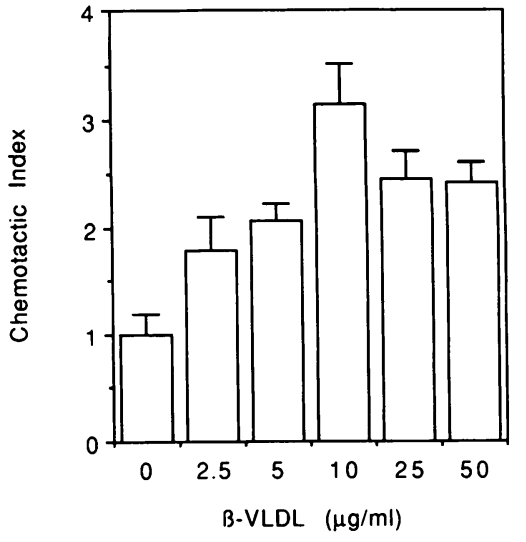

content and with the production of chemotactic activity for $\mathrm{T}$ lymphocytes. It has been previously reported that lyso-PtdCho is a chemoattractant for a murine thymic cell line and for concanavalin A-stimulated mouse spleen thymocytes (27). The concentrations of lyso-PtdCho required, however, were 20 -fold higher than the effective concentrations in the present studies and lyso-PtdCho was not effective for resting thymocytes. The reasons for the differences are not clear. Also of interest is the finding that concentrations of lyso-PtdCho increase in the artery wall early in the formation of the lesion (28). Recent work from Dr. Michael Gimbrone's laboratory has shown that lysoPtdCho can induce the expression of an endothelial cell adhesion molecule, VCAM-1, on endothelial cells (29). VCAM-1 is known to be involved in $\mathrm{T}$ lymphocyte adherence to endothelial cells (30) via a CD18-independent mechanism, and thus lyso-PtdCho may be involved in both the increased adherence and recruitment of $\mathrm{T}$ lymphocytes at sites of lesion formation.

In these studies, as in our previous studies of the monocyte chemotactic response (21), nonmetabolizable analogues of lyso-PtdCho were not chemotactic for $\mathrm{T}$ lymphocytes. This may reflect the need for further metabolism of the lyso-PtdCho in order for it to exert its chemotactic action. Alternatively, it may be that the concentration of nonmetabolizable analogues builds up in the plasma membrane and thereby erases any chemotactic gradient. Indeed, cells preincubated with the nonme-

Table III. Relationship between Extent of Oxidative Modification of LDL, LysoPC Content, and Chemotactic Properties for $T$ lymphocytes

\begin{tabular}{cccc}
\hline Time & TBARS & Lyso-PtdCho & $\mathrm{CI}$ \\
\hline$h$ & nmol $M D A / m g$ protein & nmol/10 $\mu$ g protein & mean $\pm S D$ \\
0 & 0 & 1.0 & $1.08 \pm 0.12$ \\
2 & 2 & 1.39 & $1.26 \pm 0.13$ \\
4 & 10.9 & 1.67 & $2.33 \pm 0.27$ \\
8 & 23.17 & 2.08 & $4.23 \pm 0.54$ \\
24 & 58.96 & 2.61 & $4.21 \pm 0.23$
\end{tabular}

Native LDL was oxidatively modified at a concentration of $100 \mu \mathrm{g} / \mathrm{ml}$ with $5 \mu \mathrm{M}$ copper acetate for the times indicated. These samples were then assessed at $10 \mu \mathrm{g} / \mathrm{ml}$ for their ability to chemotactically attract human $\mathrm{T}$ lymphocytes. In addition, these samples were assayed for content of TBARS, given as nanomoles MDA equivalents per milligram protein, and for lyso-PtdCho content. Values shown are means of three separate experiments.

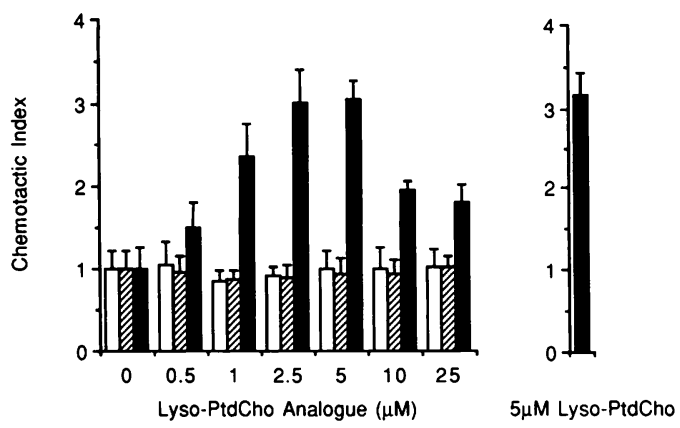

Figure 4. Human T lymphocyte response to structural analogues of lyso-PtdCho: Palmityl-ethanediol-2-phosphocholine ( $\square$ ), palmitylpropanediol-3-phosphocholine (园), and palmitoyl-ethanediol-2phosphocholine $(\square)$ were dried under nitrogen and dissolved in HBSS before being tested for chemotactic activity. The $\mathrm{CI}$ for each sample is expressed as mean $\pm \mathrm{SD}$ (seven fields counted). $5 \mu \mathrm{M}$ lyso-PtdCho was used as a positive control in each experiment. Results are representative of those from two separate experiments.

tabolizable analogues showed a significantly reduced ability to respond chemotactically to lyso-PtdCho (data not shown).

The role of the T lymphocyte in atherosclerotic lesion development has yet to be established. Recent studies show that the T cells in the lesion are polyclonal ( 31 ) and that they are in an activated state $(22,32)$. It is not clear, however, if they are recruited in an activated state or are activated locally in the lesion. Our laboratory, however, has recently demonstrated the presence of immune complexes in the artery wall, providing at least one mechanism for the local activation of $T$ cells (YläHerrtuala, S. W. Palinski, S. W. Butler, S. Picard, D. Steinberg, and J. L. Witztum, manuscript submitted for publication). Once activated, T cells are a source of a wide range of lymphokines with the potential for myriad effects on the other cellular components of the lesion (33). Activated T cells are one of the few sources of IL-4, one of the few agents capable of inducing the expression of 15-lipoxygenase in monocyte/macrophages (34). There are several lines of evidence suggesting that the oxidation of LDL by macrophages and endothelial cells can be attributed, in part, to the activity of 15-lipoxygenase (35). The evidence includes the demonstration that the gene for 15-lipoxygenase is actively expressed in macrophage-rich areas of diffuse intimal thickening (36). IL-4 is chemotactic for macrophages (37). If it is also chemotactic for monocytes, there could be an interaction in which $T$ cells help recruit additional macrophages, and vice versa. Furthermore, lymphocytes themselves have recently been shown capable of oxidizing LDL (38). Thus, there could be an important interplay between $\mathrm{T}$ lymphocytes and macrophages, accelerating both the oxidative modification of LDL and the recruitment of additional $\mathrm{T}$ lymphocytes and monocytes.

While there are, as just outlined, several ways in which $T$ lymphocyte-macrophage interactions might be proatherogenic, the situation is decidedly more complicated. This became clear from recent in vivo studies. Rats depleted of $T$ lymphocytes, either athymic rats or normal rats injected with a monoclonal antibody against $T$ cells, developed larger proliferative lesions after balloon injury than did control rats with normal $\mathrm{T}$ cell populations (39). This suggests an antiatherogenic role for $\mathrm{T}$ lymphocytes. In contrast, rats injected with cyclosporin $\mathrm{A}$, an inhibitor of $\mathrm{T}$ cell activation, were found to de- 
velop smaller lesions after balloon injury (40). It is not clear, however, if the cyclosporin A itself had a direct effect on other components of the lesion. Thus, $T$ cells could be, in principle, either proatherogenic or antiatherogenic. Only further in vivo experiments will be able to assign relative importance to the several possibilities suggested by studies in vitro.

\section{Acknowledgments}

The authors would like to thank Dr. J. Trotter (The Salk Institute, La Jolla, CA) for FACS ${ }^{\circledR}$ analysis of isolated T lymphocytes.

This work was done during the tenure of a research fellowship from the American Heart Association, California Affiliate (HFM), and was supported by National Heart, Lung and Blood Institute grant HL14197 (Specialized Center of Research on Arteriosclerosis).

\section{References}

1. Poole, J. C. F., and H. W. Florey. 1958. Changes in the endothelium of the aorta and the behaviour of macrophages in experimental atheroma of rabbits. $J$. Pathol. Bacteriol. 75:245-252.

2. Gerrity, R. G. 1981. The role of the monocyte in atherogenesis. I. Transition of blood-borne monocytes into foam cells in fatty lesions. Am. J. Pathol. 103:181-190.

3. Aqel, N. M., R. Y. Ball, H. Waldmann, and M. J. Mitchinson. 1984. Monocytic origin of foam cells in human atherosclerotic plaques. Atherosclerosis. 53:265-271.

4. Jonasson, L., J. Holm, O. Skalli, G. Bondjers, and G. K. Hansson. 1986. Regional accumulations of $\mathrm{T}$ cells, macrophages, and smooth muscle cells in the human atherosclerotic plaque. Arteriosclerosis. 6:131-138.

5. Gown, A. M., T. Tsukada, and R. Ross. 1986. Human atherosclerosis. II. Immunocytochemical analysis of the cellular composition of human atherosclerotic lesions. Am. J. Pathol. 125:191-207.

6. Munro, J. M., J. D. VanDerWalt, C. S. Munro, and E. L. Cox. 1987. An immunohistochemical analysis of human aortic fatty streaks. Hum. Pathol. 18:375-380.

7. Hansson, G. K., L. Jonasson, B. Lojsthed, S. Stemme, O. Kocher, and G. Gabbiani. 1988. Localization of T lymphocytes and macrophages in fibrous and complicated human atheroscleotic plaques. Atherosclerosis. 72:135-141.

8. Emeson, E. E., and A. L. Robertson. 1988. T lymphocytes in aortic and coronary intimas: their potential role in atherogenesis. Am. J. Pathol. 130:369376 .

9. Hansson, G. K., P. S. Seifert, G. Olsson, and G. Bondjers. 1991. Immunohistochemical detection of macrophages and $\mathrm{T}$ lymphocytes in atherosclerotic lesions of cholesterol-fed rabbits. Arterioscler. Thromb. 11:745-750.

10. Quinn, M. T., S. Parthasarathy, L. G. Fong, and D. Steinberg. 1987 Oxidatively modified low density lipoproteins: a potential role in recruitment and retention of monocyte/macrophages during atherogenesis. Proc. Natl. Acad. Sci. USA. 84:2995-2998.

11. Quinn, M. T., S. Parthasarathy, and D. Steinberg. 1988. Lysophosphatidylcholine: a chemotactic factor for human monocytes and its potential role in atherogenesis. Proc. Natl. Acad. Sci. USA. 85:2805-2809.

12. Julius, M. H., E. Simpson, and L. A. Herzenberg. 1973. A rapid method for the isolation of functional thymus-derived murine lymphocytes. Eur. J. Immunol. 3:645-649.

13. Buonassisi, V., and J. C. Venter. 1976. Hormone and neurotransmitter receptors in an established vascular endothelial cell line. Proc. Natl. Acad. Sci. USA. 73:1612-1616.

14. Havel, R. J., H. A. Eder, and J. H. Bragdon. 1955. The distribution and chemical composition of ultracentrifugally separated lipoproteins in human serum. J. Clin. Invest. 34:1345-1353.

15. Lowry, O. H., N. J. Rosebrough, A. L. Farr, and R. J. Randall. 1951. Protein measurement with the Folin phenol reagent. J. Biol. Chem. 193:265275.

16. Quinn, M. T., S. Parthasarathy, and D. Steinberg. 1985. Endothelial cellderived chemotactic activity for mouse peritoneal macrophages and the effects of modified forms of low density lipoproteins. Proc. Natl. Acad. Sci. USA. 82:59495953.
17. Parthasarathy, S., M. T. Quinn, D. C. Schwenke, T. E. Carew, and D. Steinberg. 1989. Oxidative modification of $\beta$-VLDL: potential role in monocyte recruitment and foam cell formation. Arteriosclerosis. 9:398-404.

18. Bligh, E. G., and W. J. Dyer. 1959. A rapid method of total lipid extraction and purification. Can. J. Biochem. Physiol. 37:911-917.

19. Marinetti, G. V. 1962. Chromatographic separation, identification and analysis of phosphatides. J. Lipid Res. 3:1-20.

20. Murari, R., A. El-Rahman, Y. Wedmid, S. Parthasarathy, and W. J. Baumann. 1982. Carbon-13 nuclear magnetic resonance spectroscopy of phospholipids in solution: spectral and stereochemical assignments based on ${ }^{13} \mathrm{C}-{ }^{31} \mathrm{P}$ and ${ }^{13} \mathrm{C}-{ }^{14} \mathrm{~N}$ couplings. J. Org. Chem. 47:2158-2168.

21. Quinn, M. T., N. Kondratenko, and S. Parthasarathy. 1991. Analysis of the monocyte chemotactic response to lysophosphatidylcholine: role of lysophospholipase C. Biochim. Biophys. Acta. 1082:293-302.

22. Stemme, S., J. Holm, and G. Hansson. 1992. T lymphocytes in human atherosclerotic plaques are memory cells expressing CD45RO and the integrin VLA-1. Arterioscler. Thromb. 12:206-211.

23. Miossec, P., D. Cavender, and M. Ziff. 1988. Interleukin-1 derived from human endothelial cells enhances the binding and chemotactic step of $T$ lymphocyte emigration. Clin. Exp. Immunol. 73:250-254.

24. Htin-Aung. 1990. T lymphocyte motility toward IL-1 in patients with interstitial lung diseases. Bull. Chest Dis. Res. Inst. Kyoto Univ. 23:38-47.

25. Ku, G., N. S. Doherty, J. A. Wolos, and R. L. Jackson. 1988. Inhibition by probucol of interleukin 1 secretion and its implication in atherosclerosis. $\mathrm{Am}$. J. Cardiol. 62:77B-81B.

26. Frostegård, J., R. Wu, R. Giscome, G. Holm, A. K. Lefvert, and J. Nilsson. 1992. Induction of $\mathrm{T}$ cell activation by oxidized low density lipoprotein. Arterioscler. Thromb. 12:461-467.

27. Hoffman, R. D., M. Kligerman, T. M. Sundt, N. D. Anderson, and H. S. Shin. 1982. Stereospecific chemoattraction of lymphoblastic cells by gradients of lysophosphatidylcholine. Proc. Natl. Acad. Sci. USA. 79:3285-3289.

28. Portman, O. W., and M. Alexander. 1969. Lysophosphatidylcholine concentrations and metabolism in aortic intima plus inner media: effect of nutritionally induced atherosclerosis. J. Lipid Res. 10:158-165.

29. Kume, N., M. I. Cybulsky, and M. A. Gimbrone. 1992. Lysophosphatidylcholine, a component of atherogenic lipoproteins, induces mononuclear leukocyte adhesion molecules in cultured human and rabbit arterial endothelial cells. J. Clin. Invest. 90:1138-1144.

30. Carlos, T. M., B. R. Schwartz, N. L. Kovach, E. Yee, M. Rosa, L. Osborn, G. Chi-Rosso, B. Newman, R. Lobb, and M. Rosso. 1990. VCAM-1 mediates a CD18-independent pathway of lymphocyte adherence to HUVEC (stimulated with cytokines). Blood. 76:965-970.

31. Stemme, S., L. Rymo, and G. K. Hansson. 1991. Polyclonal origin of T lymphocytes in human atherosclerotic plaques. Lab. Invest. 65:654-660.

32. Hansson, G. K., J. Holm, and L. Jonasson. 1989. Detection of activated T lymphocytes in the human atherosclerotic plaque. Am. J. Pathol. 135:169-175.

33. Libby, P., and G. K. Hansson. 1991. Involvement of the immune system in human atherogenesis: current knowledge and unanswered questions. $L a b$. Invest. 64:5-15.

34. Conrad, D. J., H. Kuhn, M. Mulkins, E. Highland, and E. Sigal. 1992 Specific inflammatory cytokines regulate the expression of human monocyte 15-lipoxygenase. Proc. Natl. Acad. Sci. USA. 89:217-222.

35. Rankin, S. M., S. Parthasarathy, and D. Steinberg. 1991. Evidence for a dominant role of lypoxygenase ( $s$ ) in the oxidation of LDL by mouse peritoneal macrophages. J. Lipid Res. 32:449-456.

36. Ylä-Herttuala, S., M. E. Rosenfeld, S. Parthasarathy, E. Sigal, T. Sarkioja, J. L. Witztum, and D. Steinberg. 1991. Gene expression in macrophage-rich human atherosclerotic lesions. 15-lipoxygenase and acetyl low density lipoprotein receptor messenger RNA colocalize with oxidation specific lipid-protein adducts. J. Clin. Invest. 87:1146-1152.

37. Hiester, A. A., D. R. Metcalf, and P. A. Campbell. 1992. Interleukin-4 is chemotactic for mouse macrophages. Cell. Immunol. 139:72-80.

38. Lamb, D. J., G. M. Wilkins, and D. Leake. 1992. The oxidative modification of low density lipoprotein by human lymphocytes. Atherosclerosis. 92:187192.

39. Hansson, G. K., J. Holm, S. Holm, Z. Fotev, H. J. Hedrich, and J. Fingerle. 1991. T lymphocytes inhibit the vascular response to injury. Proc. Natl. Acad. Sci. USA. 88:10530-10534.

40. Stemme, S., L. Jonasson, J. Holm, and G. K. Hansson. 1989. Immunologic control of vascular cell growth in arterial respose to injury and atherosclerosis. Transplant. Proc. 21:3697-3699. 